\title{
Differential effects of prenatal stress and glucocorticoid administration on postnatal growth and glucose metabolism in rats
}

\author{
K L Franko, A J Forhead and A L Fowden \\ Department of Physiology, Development and Neuroscience, University of Cambridge, Downing Street, Cambridge CB2 3EG, UK \\ (Correspondence should be addressed to A L Fowden; Email: alf1000@cam.ac.uk)
}

\begin{abstract}
Glucocorticoid administration during pregnancy programmes cardiovascular and metabolic functions in the adult offspring. Often, the control procedures are stressful per se and raise maternal glucocorticoid concentrations. This study compared the effects of maternal injection with dexamethasone $(\mathrm{dex}, 200 \mu \mathrm{g} / \mathrm{kg}$ ) or saline with no treatment from 15 to 20 days of rat pregnancy on offspring growth and glucose metabolism. Near term, maternal corticosterone concentrations were higher in the saline-treated dams and lower in the dex-treated dams relative to untreated animals. In both male and female offspring, growth rate was measured for 14 weeks, and glucose tolerance was assessed between 12 and 13 weeks together with body fat content and plasma concentrations of insulin, leptin, and corticosterone between 14 and 15 weeks. Offspring liver was collected at different ages and was analyzed for glycogen content and gluconeogenic
\end{abstract}

enzyme activity. Compared with untreated animals, both dex and saline treatments altered postnatal growth although adult body weight was unaffected. The two treatments had different effects on adult insulin concentrations and on hepatic glycogen content and gluconeogenic enzyme activities both pre- and postnatally. Relative to untreated animals, adult glucose tolerance was improved by maternal saline injection in males but not in females, while it was impaired in female offspring but not in male offspring of the dex-treated dams. Adult glucose tolerance was related to male body fat content but not to female body fat content. Dex and saline treatments of pregnant rats have differential sex-linked effects on the growth and glucose metabolism of their offspring, which indicates that the programming actions of natural and synthetic glucocorticoids may differ.

Journal of Endocrinology (2010) 204, 319-329

\section{Introduction}

Human epidemiological studies have shown that impaired intrauterine growth is associated with an increased risk of cardiovascular, metabolic, and other diseases in later life (Barker 1994). These associations have led to the concept that adult disease can originate in utero as a result of developmental programming of key tissues and organ systems during suboptimal intrauterine conditions associated with poor fetal growth (Gluckman et al. 2008). Experimentally, prenatal programming of postnatal metabolism has been demonstrated in a number of species using a range of different techniques to induce intrauterine growth restriction (IUGR) including maternal stress and glucocorticoid administration (see McMillen \& Robinson 2005, Fowden et al. 2006, Seckl 2008). Similarly, in naturally occurring IUGR in polytocous species, low birth weight is associated with adult glucose intolerance and altered fat deposition (Poore \& Fowden 2002, 2004). In both naturally occurring and experimentally induced IUGR, the programmed alterations in postnatal glucose handling are associated with functional changes in a range of tissues involved in growth and glucoregulation, including several endocrine systems (see McMillen \& Robinson 2005, Fowden et al. 2006).

In pregnant rats, maternal treatment with the synthetic glucocorticoid, dexamethasone (dex), via a number of different routes leads to IUGR and glucose intolerance of the adult offspring, accompanied by changes in the liver, fat, and skeletal muscle, and in circulating concentrations of insulin, leptin, and corticosterone in the adults (Muneoka et al. 1997, Nyirenda et al. 1998, 2006, Smith \& Waddell 2000, Sugden et al. 2001, Cleasby et al. 2003, O’Regan et al. 2004, Wyrwoll et al. 2006, 2008). Similarly, stresses during rat pregnancy that raise maternal glucocorticoid concentrations, such as restraint, noise, and isolation, induce IUGR and alter glucose handling and hypothalamic-pituitary-adrenal (HPA) axis function in the adult offspring (Barbazanges et al. 1996, Vallée et al. 1996, Maccari et al. 2003, Lesage et al. 2004, D'mello \& Lin 2006). Indeed, in pregnant rats, procedures such as saline injection, often used as a control in studying prenatal origins of adult disease, lead to elevated maternal and fetal corticosterone concentrations, which may programme tissue development per se (Ward \& Weisz 1984, Barbazanges et al. 1996). Consequently, the stress of injection may mimic, 
in part, the programming effects of synthetic glucocorticoids and may mean that the comparison of offspring from dex- and saline-treated, or vehicle-treated, dams may be examining different types or degrees of intrauterine programming. However, little is known about the effects of commonly used control procedures, such as maternal saline or vehicle injection, on glucose metabolism of their offspring. The current study, therefore, compared the effects of maternal injection with dex or saline with no treatment during rat pregnancy on the growth and glucose metabolism of their male and female offspring.

\section{Materials and Methods}

\section{Animals}

A total of 32 virgin female Wistar rats aged between 12 and 15 weeks were used (Fig. 1). They were maintained at $22^{\circ} \mathrm{C}$ in a $12 \mathrm{~h}$ light: $12 \mathrm{~h}$ darkness cycle and were fed a standard laboratory chow throughout the study (Standard Breeding Diet No. 3, Special Diet Services, Essex, UK). Females were housed overnight with a male Wistar rat (minimum age 15 weeks) and were checked daily for the presence of a copulatory plug. The day after mating was taken as day 0 of pregnancy (term is 21.5 days). Before mating, all females were housed in groups, whereas after mating, they were housed individually to allow the measurement of daily food intake. Dams were weighed on days 0,15 , and 20 of pregnancy.

\section{Experimental procedures}

All experimental procedures were carried out under the Animals (Scientific Procedures) Act 1986. On days 15-19 of pregnancy inclusive, 22 dams were injected subcutaneously with either saline $(400 \mu \mathrm{l}, 0.9 \% \mathrm{w} / \mathrm{v}, n=11)$ or dex (200 $\mu \mathrm{g} / \mathrm{kg}$, dex 21-phosphate sodium salt, Sigma, in $400 \mu \mathrm{l}$ saline, $n=11$, Fig. 1). Administration of dex to pregnant rats at this dose and stage of gestation is known to programme cardiovascular and metabolic functions in the adult offspring (Nyirenda et al. 1998, Cleasby et al. 2003, Drake et al. 2004, O'Regan et al. 2004). Ten dams acted as controls and received no treatment (Fig. 1). Between 0800 and $1000 \mathrm{~h}$ on day 20, a subset of each group ( $n=6$ control, $n=7$ saline, and $n=7$ dex dams) was anesthetized with a mixture of sodium pentobarbitone and chloral hydrate (equithesin, $0.6 \mathrm{ml} / 100 \mathrm{~g}$ body weight, i.p., and $9 \cdot 6 \%$ pentobarbital sodium, BDH Chemical Ltd, Poole, England; $42 \cdot 6 \%$ chloral hydrate, Sigma-Aldrich $\mathrm{Co}$ ). Once anesthetized, a ventral incision was made in the abdomen and a 2-ml blood sample was collected from the uterine vein for the measurement of blood glucose and plasma corticosterone concentrations (Fig. 1). Fetuses were removed individually from each horn, weighed, and killed by decapitation immediately after delivery. A small blood sample $(<20 \mu \mathrm{l})$ was obtained from the severed vessels in the fetal neck for the measurement of blood glucose concentrations. The fetal liver was removed, weighed, and then flash-frozen in liquid nitrogen for subsequent analyses of glycogen content and gluconeogenic enzyme activities (Franko et al. 2009). When all fetuses were delivered, the dam was killed with a lethal dose of anesthetic $(200 \mathrm{mg} / \mathrm{kg}$ sodium pentobarbitone, i.v., Dolethal, Vetoquinol UK Ltd, Buckingham, UK). Maternal blood samples were centrifuged at $4{ }^{\circ} \mathrm{C}$, and the plasma was stored at $-20^{\circ} \mathrm{C}$ until the measurement of corticosterone concentrations.

The remaining four dams in each of the saline and dex groups received another injection on day 20 and were then allowed to deliver naturally without further treatment,

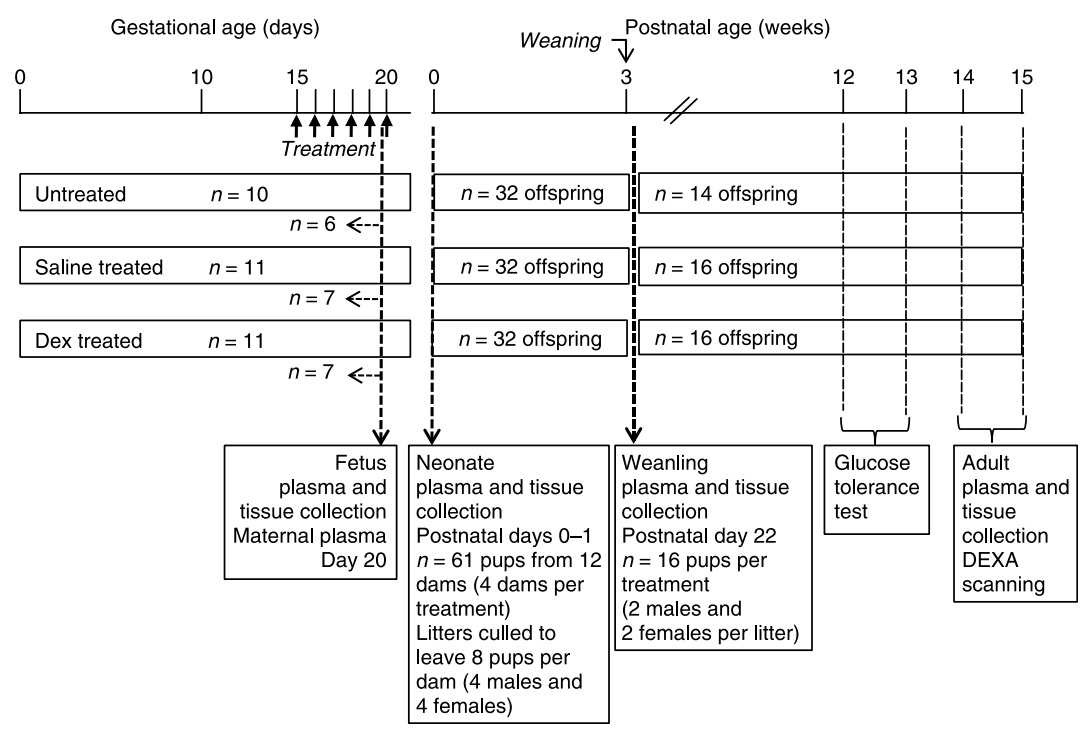

Figure 1 Schematic diagram of the experimental protocol indicating the sequence of experimental procedures and the numbers of dams and offspring involved at each stage of the experiment. 
together with the four remaining control dams (Fig. 1). On day 1 of postnatal life, all litters were culled to eight pups so that four females and four males nearest to the median weight for the litter were reared by their mother (Fig. 1). The rest of each litter was killed by decapitation (Fig. 1). A small blood sample $(<20 \mu \mathrm{l})$ was taken from the severed neck vessels to measure blood glucose, and the liver from culled neonates was collected into liquid nitrogen. Reared pups were weighed weekly until 21 days of postnatal age when they were weaned, and then on day 22, half of each litter (two males and two females) were selected randomly for culling by cervical dislocation. A blood sample $(<20 \mu \mathrm{l})$ and the liver were collected from each of the culled weanlings $(n=16$ controls, $n=16$ saline, and $n=16$ dex offspring from 4 dams per treatment, Fig. 1). The remaining two males and two females from each litter were individually marked and fed standard laboratory chow (Standard Breeding Diet No. 3, Special Diet Services) until the end of experimental procedures between 14 and 15 weeks of postnatal age (Fig. 1). They were housed in groups by sex with a control, saline-treated individual, and dex-treated individual in each cage. Offspring were weighed weekly from weaning to 14 weeks for the calculation of fractional growth rate (FGR).

Between postnatal weeks 12 and 13, a glucose tolerance test was carried out after an overnight fast $(n=14$ control, $n=16$ saline, and $n=16$ dex from 4 dams per treatment, Fig. 1). Glucose $(0 \cdot 2 \mathrm{~g} / \mathrm{kg}$ as a $20 \%$ solution) was injected intraperitoneally, and blood samples $(<20 \mu \mathrm{l})$ for the measurement of blood glucose concentrations were collected from a single nick in the tail vein at -15 and $0 \mathrm{~min}$ before and at 15,30 , $45,60,90$, and $120 \mathrm{~min}$ after glucose administration. At the end of the glucose tolerance test, the rats were returned to their cages until between 14 and 15 weeks when they were weighed and euthanized by cervical dislocation in the fed state for the collection of blood $(2 \mathrm{ml})$ and liver samples (Fig. 1). After removal of the liver, the fat content of the abdominal region of the carcass was measured using dual emission X-ray absorption (DEXA) scanning (LUNAR PIXImus Densitometer, GE Lunar Corporation, Madison, WI, USA). The scan included peritoneal, omental, mesenteric, perirenal, and subcutaneous fat deposits in the abdominal cavity. Thereafter, the discrete peritoneal and perirenal fat deposits were excised and weighed.

\section{Biochemical analyses}

Blood glucose concentrations were measured using a handheld glucometer (Lifespan, Ortho-Clinical Diagnostics, High Wycombe, UK). Concentrations of insulin, leptin, and corticosterone were measured in adult plasma by RIA using commercially available kits (insulin and leptin, Linco Research, Saint Charles, MO, USA; corticosterone, ImmuChem, Orangeburg, NY, USA). For each hormone, all samples were analyzed in a single assay. The lower limits of sensitivity of the assay for insulin, leptin, and corticosterone were $0 \cdot 1,0 \cdot 06$, and $10 \mathrm{ng} / \mathrm{ml}$ respectively. The intra-assay coefficient of variation was $\leq 10 \%$ for all three assays. The hepatic glycogen content and activities of glucose-6phosphatase (G6Pase, EC 3.1.3.9) and phosphoenolpyruvate carboxykinase (PEPCK, EC 4.1.1.49) were measured in the liver from the postnatal offspring and from, at least, four fetuses from each litter (two of each sex) using established methods described previously (Franko et al. 2007, 2009). Briefly, G6Pase activity was measured as phosphate production from glucose-6-phosphate, while total hepatic PEPCK activity was estimated by measuring ${ }^{14} \mathrm{C}$ incorporation into malate (Fowden et al. 1993). For both gluconeogenic enzymes, tissue was homogenized in ice-cold sucrose $(0.25 \mathrm{M})$ and activity was measured over a $10-$ min period at $37^{\circ} \mathrm{C}$. Hepatic glycogen content was measured as glucose produced by amyloglucosidase activity in $10 \mathrm{~min}$ at $55^{\circ} \mathrm{C}$ (Franko et al. 2007). The inter-assay coefficients of variation of a fetal liver homogenate in the glycogen, G6Pase, and PEPCK assays were $9,11 \cdot 8$, and $5 \%$ respectively. Hepatic protein content was determined using the Lowry assay. All tissue and plasma analyses were done in duplicate. Measurements of the fat content of the region of interest by DEXA scanning were validated biochemically in ten additional Wistar rats using the chloroform/methanol method of fat extraction (Folch et al. 1957). Body fat content measured by DEXA scanning was linearly related to the fat content determined biochemically as follows: $y=0 \cdot 79 x$ $+1 \cdot 11, n=10, r=0 \cdot 955$, and $P<0 \cdot 01$, where $y$ is the percentage fat content measured by DEXA scanning and $x$ is the percentage fat content determined biochemically.

\section{Statistical analyses}

Means ( \pm s.E.M.) are presented throughout. Tissue and blood samples were collected at four ages: fetuses, neonates, weanlings, and adults between 14 and 15 weeks of postnatal age. The effect of treatment in the pregnant dams was assessed by one-way ANOVA with the Holm-Sidak post hoc test (Sigmastat 3.5; Systat Software Inc., Point Richmond, CA, USA). For the offspring, the effects of treatment and sex were assessed by linear mixed model using the Sidak post hoc test using the dam as a subject with each offspring and time, or age, as repeated measures as appropriate (SPSS 16.0, SPSS Inc., Chicago, IL, USA). When sex of the offspring or an interaction between maternal treatment and sex of the offspring was identified as a significant influence, male and female data were analyzed separately by linear mixed model using the Sidak post hoc test. Associations between the adult variables were assessed by linear mixed model using generalized estimating equations clustered on the dam to allow for nonindependence of littermates (Stata v10.1, Statacorp., College Station, TX, USA). FGR from birth to weaning and from weaning to 14 weeks was calculated for each animal as weight increment over the entire period expressed as grams gained per week per gram starting weight at birth or weaning respectively. 


\section{Results}

Pregnancy outcome

Dam weight was not significantly different between the groups on days 0 and 15 of pregnancy, but by day 20 , it was significantly lower in the dex-treated animals than in the control animals (Table 1). Maternal weight gain was, therefore, similar in the three groups from 0 to 15 days, but relative to the controls, it was significantly less in the salineand dex-treated groups during the treatment period (Table 1). Weight gain over the treatment period was also significantly less in the dex-treated dams than in the saline-treated dams (Table 1). Maternal food intake did not differ between groups before or during the treatment period (Table 1). Litter size was also similar in the three groups (Table 1). On day 20, maternal concentrations of plasma corticosterone were highest in dams treated with saline and lowest in those receiving dex (Table 1). There were no significant differences in maternal blood glucose concentrations with treatment on day 20 (Table 1).

\section{Offspring growth and morphometry}

Compared with the controls, dex treatment reduced body weight of the offspring in utero, at birth, during suckling, and for 4 weeks after weaning $(P<0 \cdot 01$, all cases, Fig. 2). Body weight of offspring from the saline-treated dams was similar to that of controls in utero, intermediate between the values of the control and dex-treated groups at birth (Fig. 2A), and significantly less than control values during suckling and from weaning to 7 weeks $(P<0 \cdot 01$, all cases, Fig. $2 \mathrm{~B}$ and $\mathrm{C})$.

Table 1 Mean ( \pm s.E.M.) food intakes and absolute and incremental $(\Delta)$ body weights at different periods of pregnancy, litter size, and concentrations of blood glucose and plasma corticosterone on day 20 of pregnancy in untreated, control rat dams $(n=10$ dams or for corticosterone and glucose $n=6$ dams) and those treated with saline or dexamethasone from days 15 to 19 of pregnancy ( $n=11$ dams or for corticosterone and glucose $n=7$ dams in each treated group)

\begin{tabular}{|c|c|c|c|}
\hline & Control & Saline & Dexamethasone \\
\hline \multicolumn{4}{|c|}{ Food intake (g/day per rat) } \\
\hline $1-10$ days & $21 \cdot 1 \pm 0 \cdot 6$ & $20 \cdot 4 \pm 0 \cdot 9$ & $21 \cdot 6 \pm 1 \cdot 0$ \\
\hline $11-15$ days & $25 \cdot 0 \pm 1 \cdot 1$ & $22 \cdot 5 \pm 1 \cdot 1$ & $25 \cdot 3 \pm 1 \cdot 0$ \\
\hline 16-20 days & $24 \cdot 9 \pm 0 \cdot 7$ & $24 \cdot 2 \pm 1 \cdot 1$ & $24 \cdot 7 \pm 1 \cdot 2$ \\
\hline \multicolumn{4}{|l|}{ Body weight (g) } \\
\hline Day 0 & $260 \pm 11$ & $258 \pm 10$ & $268 \pm 10$ \\
\hline Day 15 & $312 \pm 11$ & $305 \pm 12$ & $315 \pm 13$ \\
\hline Day 20 & $388 \pm 10^{\mathrm{a}}$ & $364 \pm 14^{a, b}$ & $347 \pm 15^{\mathrm{b}}$ \\
\hline$\Delta 0-15$ days & $52 \pm 6$ & $47 \pm 3$ & $46 \pm 4$ \\
\hline$\Delta 15-20$ days & $76 \pm 8^{a}$ & $59 \pm 4^{b}$ & $31 \pm 4^{\mathrm{c}}$ \\
\hline Litter size & $13 \cdot 4 \pm 0 \cdot 7$ & $13 \cdot 9 \pm 0.5$ & $14 \cdot 3 \pm 0 \cdot 7$ \\
\hline Corticosterone (ng/ml) & $652 \pm 59^{a}$ & $818 \pm 52^{b}$ & $465 \pm 25^{c}$ \\
\hline Glucose $(\mathrm{mmol} / \mathrm{l})$ & $5 \cdot 00 \pm 0 \cdot 61$ & $4 \cdot 90 \pm 0.9$ & $4 \cdot 33 \pm 0 \cdot 42$ \\
\hline
\end{tabular}

Values within rows with different letters as superscripts are significantly different from each other $(P<0 \cdot 01$, one-way ANOVA).
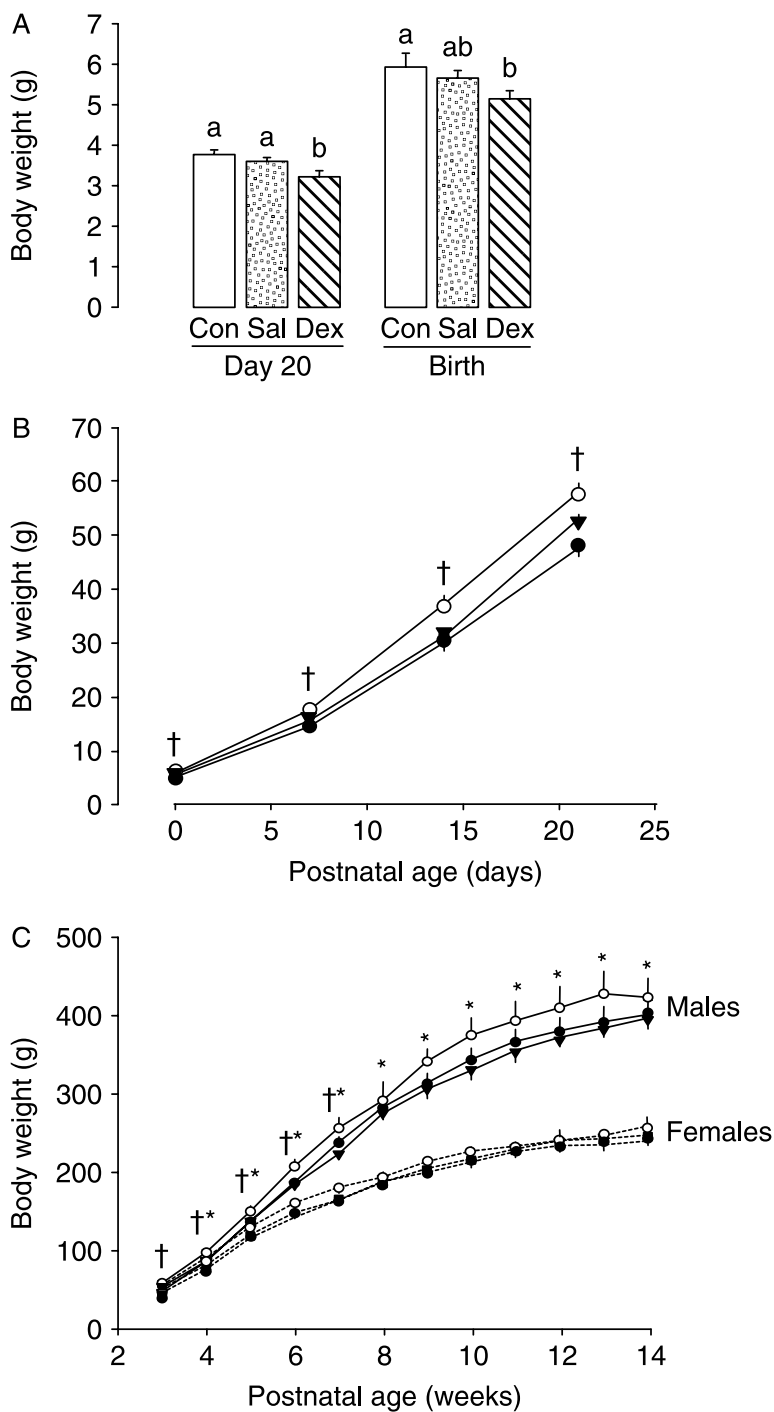

Figure 2 Mean ( \pm S.E.M.) body weights of the offspring of untreated, control rat dams, and dams treated with saline or dexamethasone from day 15 to day 19 (fetuses) or day 20 of pregnancy (all other offspring) (A) on day 20 of pregnancy and at birth (Con, open columns; Sal, stippled columns; Dex, striped columns $n=4-7$ I per treatment at each age), (B) during suckling from birth to weaning (Con, open circles; Sal, triangles; Dex, filled circles, $n=4$ I per treatment group with four male and four female pups in each litter), and $(\mathrm{C})$ in males (solid lines) and females (dashed lines) from weaning to 14 weeks of postnatal age (Con, open circles, $n=7$ males and $n=7$ females from four dams; Sal, triangles, $n=8$ males and $n=8$ females from four dams; Dex, filled circles, $n=8$ males and $n=8$ females from four dams). At each age in (A), columns with different letters are significantly different from each other $(P<0 \cdot 01)$, while columns sharing common letters are not significantly different from each other $(P>0 \cdot 05$, linear mixed model). In (B) and (C), significant effects of maternal treatment $\left({ }^{\dagger} P<0 \cdot 01\right)$ and/or sex of the offspring $\left({ }^{*} P<0 \cdot 01\right)$ are shown by linear mixed model. There were no significant effects of sex of the offspring on body weight in $(\mathrm{A})$ or $(\mathrm{B})$. 
By postnatal week 8 , maternal treatment no longer had a significant influence on offspring body weight (Fig. 2C). Body weights of male and female offspring from the salineand dex-treated dams, although still numerically smaller, were not significantly different from those of the controls at the time of the glucose tolerance test or at tissue collection (Table 2). Sex of the offspring had no significant effect on the body weight of fetal, newborn, or weanling pups $(P>0 \cdot 05$, all cases). However, by postnatal week 4 , males weighed more than females and remained heavier thereafter (Fig. 2C). There was no interaction between maternal treatment and sex of the offspring in determining body weight at any of the ages studied $(P>0 \cdot 05$, all cases, Fig. 2C, Table 2$)$.

FGR, calculated as weight increment from birth to weaning, was unaffected by maternal treatment or sex of the offspring $(P>0 \cdot 05$, both cases, Table 2$)$. In contrast, FGR from weaning to 14 weeks was influenced by both maternal treatment and sex of the offspring $(P<0 \cdot 05$, both cases, Table 2). Over this period, FGR was significantly less in females than in males (Table 2). In males, postweaning FGR was higher in offspring of the dex-treated dams than in the other groups with no significant difference between the control and saline-treated groups (Table 2). In females, maternal treatment had no effect on the postweaning FGR (Table 2).

In adults between 14 and 15 weeks, the fat content of the abdominal region measured by DEXA scanning was influenced by both maternal treatment $(P<0.02)$ and sex of the offspring $(P<0 \cdot 001)$. Females had less fat than males irrespective of treatment (Table 2). In the males, fat content was significantly lower in offspring of the saline-treated animals than in the other groups $(P<0 \cdot 05$, Table 2$)$. A similar trend was observed in the females, but this did not reach statistical significance $(P>0 \cdot 05$, Table 2$)$. When perirenal and peritoneal fat contents were calculated gravimetrically, the values were influenced by maternal treatment $(P<0 \cdot 02)$ and sex of the offspring $(P<0 \cdot 05)$, with significantly lower values in the dex-treated group than in the control group $(P<0 \cdot 02)$. This effect of dex appeared to be more pronounced in males than in females (Table 2). When data from all adult animals were combined, there were significant positive relationships between the DEXA percentage fat content and postweaning FGR $(P<0 \cdot 001, n=46$ offspring from 12 dams $)$ when differences in current weight were taken into account. Percentage fat content calculated gravimetrically was not related to postweaning FGR $(P>0 \cdot 05, n=46$ offspring from 12 dams).

\section{Plasma hormone concentrations}

Between 14 and 15 weeks, plasma leptin concentrations were significantly lower, while plasma corticosterone concentrations were significantly higher in females than in males irrespective of treatment (Table 2). Neither of these hormone concentrations was affected by maternal treatment (Table 2). In contrast, plasma insulin concentrations were influenced by both maternal treatment and sex of the offspring with lower values in females than in males (Table 2). Insulin levels were significantly higher in the saline-treated group than in the dex-treated group with intermediate values in the controls $(P<0 \cdot 02$, Table 2$)$. When data from all adult offspring were combined, plasma leptin concentrations were positively related to DEXA fat content $(P<0 \cdot 016, n=32$ offspring from 11 dams). Conversely, plasma corticosterone concentrations were inversely related to DEXA fat content $(P<0 \cdot 012, n=40$ offspring from 12 dams $)$. In contrast, insulin concentrations were unrelated to DEXA fat content $(P>0 \cdot 05)$, but were positively related to postweaning FGR $(P<0 \cdot 025, n=35$ offspring from 12 dams). No significant relationships were observed between any of these hormone concentrations and percentage fat content calculated gravimetrically $(P>0 \cdot 05$, all cases). Nor were any of these hormone concentrations related to fat content (DEXA or excised) when males and females were considered separately $(P>0 \cdot 05$, all cases, $n=16-20$ offspring from 12 dams).

\section{Glucose metabolism}

Glucose concentrations At all ages studied, blood glucose concentrations in the fed state were unaffected by maternal treatment or sex of the offspring $(P>0 \cdot 05)$. The mean blood glucose concentrations in the fetuses, neonates, and weanlings were $4 \cdot 3 \pm 0 \cdot 2 \mathrm{mmol} / 1 \quad(n=19$ litters $), \quad 3 \cdot 9 \pm 0 \cdot 2 \mathrm{mmol} / 1$ ( $n=12$ litters), and $8 \cdot 9 \pm 0 \cdot 2 \mathrm{mmol} / 1$ ( $n=43$ pups from 12 dams) respectively. Adult glucose concentrations in the fasted state were significantly less than those in the fed state and were also unaffected by maternal treatment or sex of the offspring (Table 2).

Hepatic glycogen content and gluconeogenic enzyme activities Hepatic glycogen content and activities of G6Pase and PEPCK were not affected by sex of the offspring at any of the ages studied $(P>0.05$, all cases). Maternal dex treatment influenced hepatic glycogen content in the fetuses but not in the other groups of offspring (Fig. 3A). In fetuses, hepatic glycogen content was highest in the dex-treated group and lowest in the controls with intermediate values in the saline-treated group (Fig. 3A). A similar pattern of hepatic G6Pase activity with maternal treatment was observed in the fetuses (Fig. 3B). However, G6Pase activity was unaffected by maternal treatment at any of the postnatal ages studied (Fig. 3B). In contrast, hepatic PEPCK activity differed with maternal treatment at all ages studied, although the specific effects of treatment varied at each age (Fig. 3C). In fetuses, hepatic PEPCK activity was significantly higher in the treated group than in the untreated group, but it was not significantly different between saline and dex treatments (Fig. 3C). At birth, the saline-treated group had a lower PEPCK activity than the other two groups, while in weanlings, PEPCK activity was lowest in controls and highest in the dex-treated group with intermediate values in the saline-treated group (Fig. 3C). In adults, hepatic PEPCK 


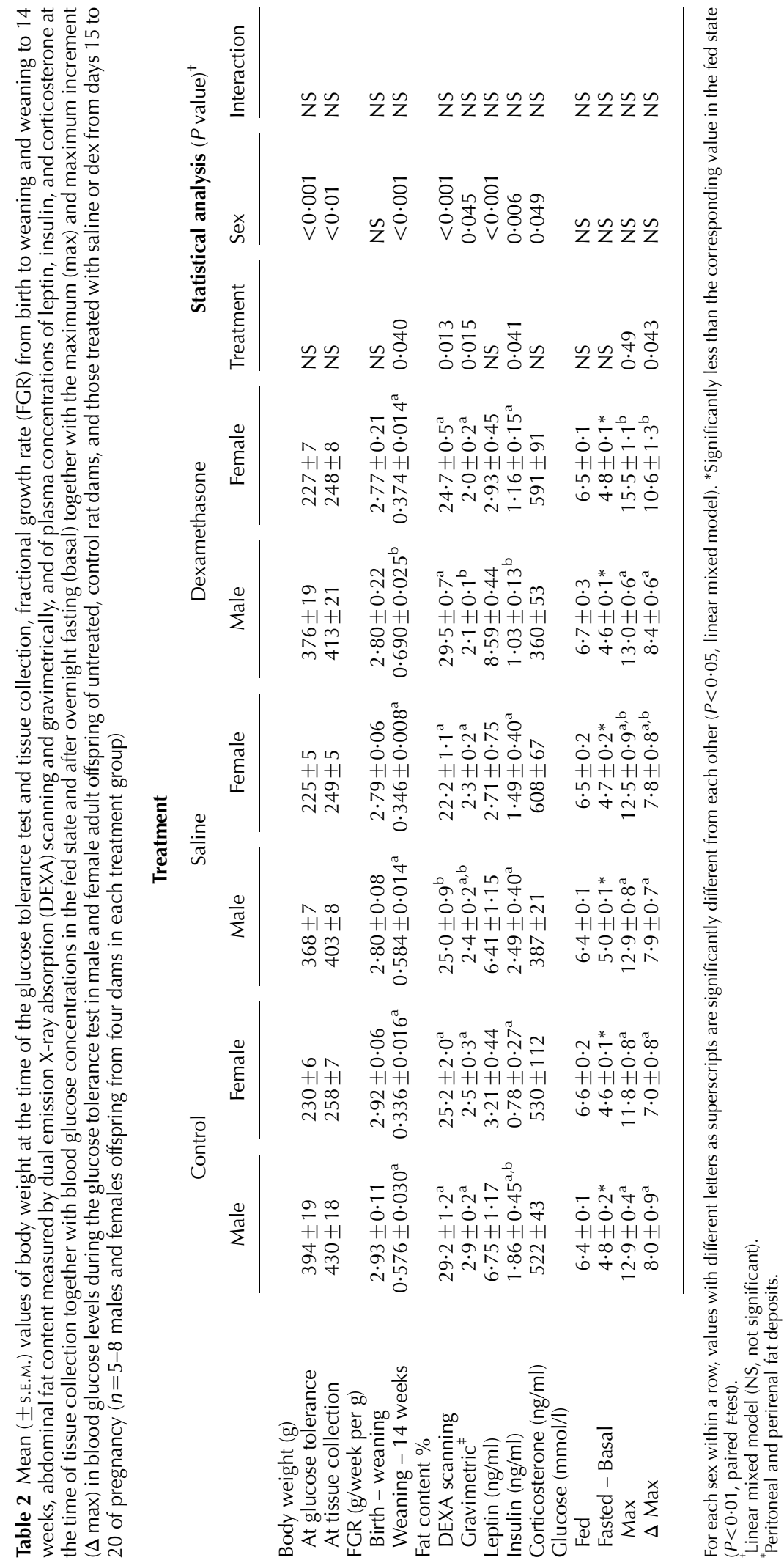



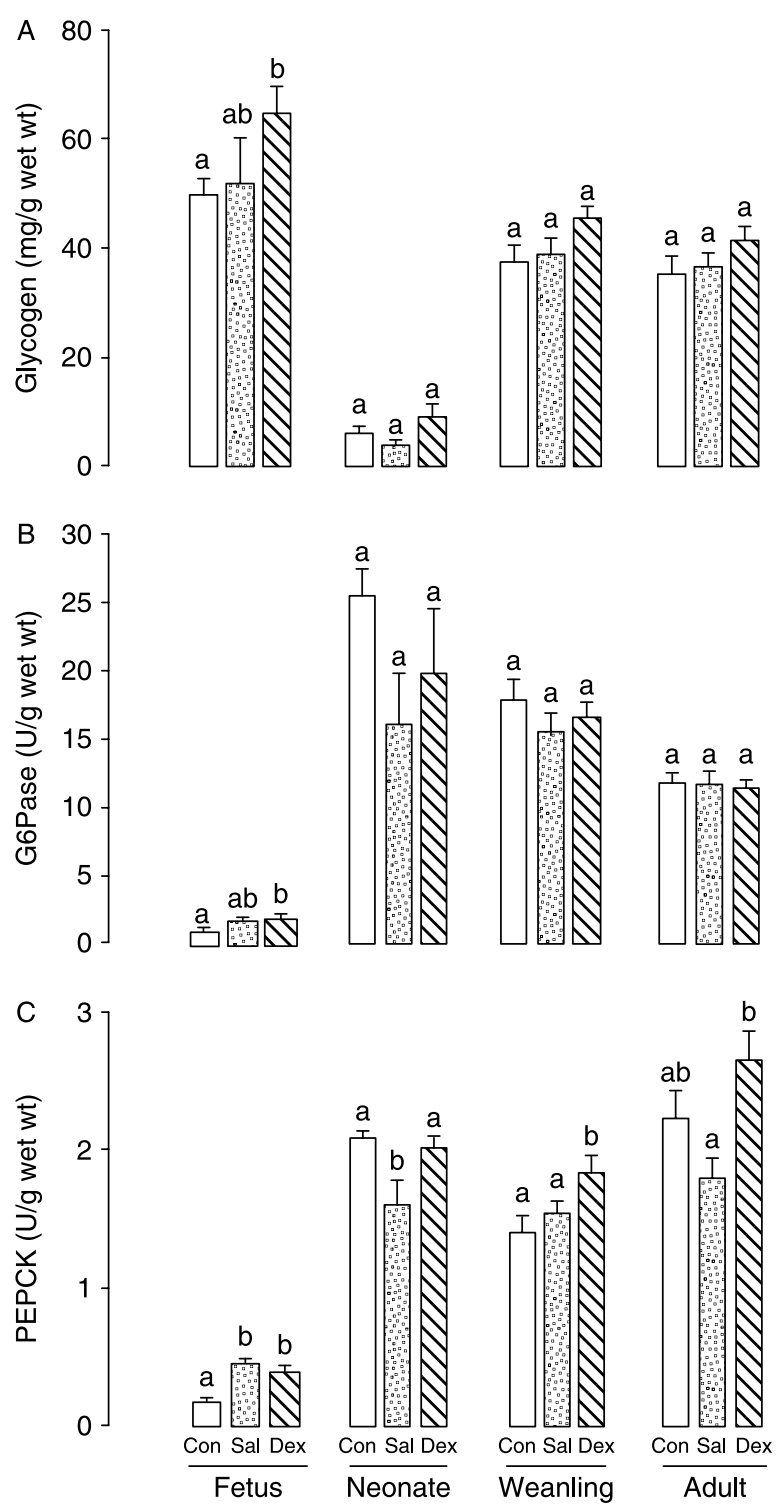

Figure 3 Mean ( \pm S.E.M.) values of (A) glycogen content, (B) glucose-6-phosphatase (G6Pase) activity, and (C) phosphoenolpyruvate carboxykinase (PEPCK) activity of liver from the fetuses, neonates, weanlings, and adult offspring of untreated control dams (Con, open columns) or dams treated with saline (Sal, stippled columns) or dexamethasone (Dex, striped columns) from day 15 to day 19 (fetuses) or day 20 of pregnancy (all other offspring). For the fetuses and neonates, $n=4-7$ I for each treatment group. For weanlings and adults, $n=14-16$ individual offspring per treatment group with four dams per treatment. At each age, columns with different letters are significantly different from each other $(P<0 \cdot 02)$, while columns sharing common letters are not significantly different from each other $(P>0 \cdot 05$, linear mixed model). In (A), (B), and (C), there were no significant effects of sex of the offspring at any age studied $(P>0 \cdot 05$, linear mixed model).

activity was higher in the dex-treated group than in the saline-treated group, but neither of these values was significantly different from that in the controls (Fig. 3C).

At all ages studied, hepatic protein content was unaffected by either maternal treatment or sex of the offspring $(P>0 \cdot 05$, data not shown).

Adult glucose tolerance Adult glucose tolerance was affected by maternal treatment $(P<0 \cdot 003)$ but not by sex of the offspring $(P>0 \cdot 05$, Fig. 4). In all animals irrespective of sex, maternal treatment influenced the glucose concentrations observed 15, 30, and $45 \mathrm{~min}$ after glucose administration (Fig. 4Ai). The maximum concentration and increment in blood glucose differed with maternal treatment but not with sex of the offspring (Table 2). For all animals, the area under the glucose curve (AUGC) was smallest for the saline-treated group and significantly less in the saline-treated animals than in the dex-treated animals with intermediate values in the controls (Fig. 4Aii). However, there was an interaction between maternal treatment and sex of the offspring in determining the time profile of the glucose concentration and the areas under the curve such that the specific effects of treatment depended on sex of the offspring $(P<0 \cdot 05$, all cases, Table 2). In males, glucose levels were significantly different with treatment 30 and $45 \mathrm{~min}$ after glucose administration, and AUGC was significantly less in the saline-treated group than in either of the other two groups (Fig. 4Bi and ii). No significant difference was observed in AUGC between male offspring of the control and dex-treated dams (Fig. 4Bii). There were also no significant differences in the maximum concentration or increment in blood glucose with maternal treatment in male offspring (Table 2). In contrast, in females, blood glucose concentrations were significantly different with treatment at 15 and $30 \mathrm{~min}$, and AUGC was significantly greater in the dex-treated group than in the other two groups (Fig. 4Ci and ii). Unlike in males, there was no significant difference in AUGC between female offspring of the control and saline-treated dams (Fig. 4Cii). The maximum concentrations and increment in blood glucose in females were also significantly higher in the dextreated group than in the control group in contrast to the males (Table 2).

There were no significant correlations between AUGC and current body weight, postweaning FGR, body fat content (DEXA or excised), hepatic glycogen content, or activities of G6Pase and PEPCK when data from all the adults were combined $(P>0 \cdot 05$, all cases). In males, AUGC was positively correlated to DEXA fat content $(P<0 \cdot 01, n=23$ offspring from $11 \mathrm{dams})$, but not to any of the other variables $(P>0 \cdot 05$, all other cases). In females, AUGC was positively correlated to postweaning FGR $(P<0 \cdot 041, n=22$ offspring from 12 dams) and was unrelated to any of the other variables $(P>0 \cdot 05$, all other cases).

\section{Discussion}

The results demonstrate that dex treatment and the stress associated with saline injection of rats during late pregnancy 
A
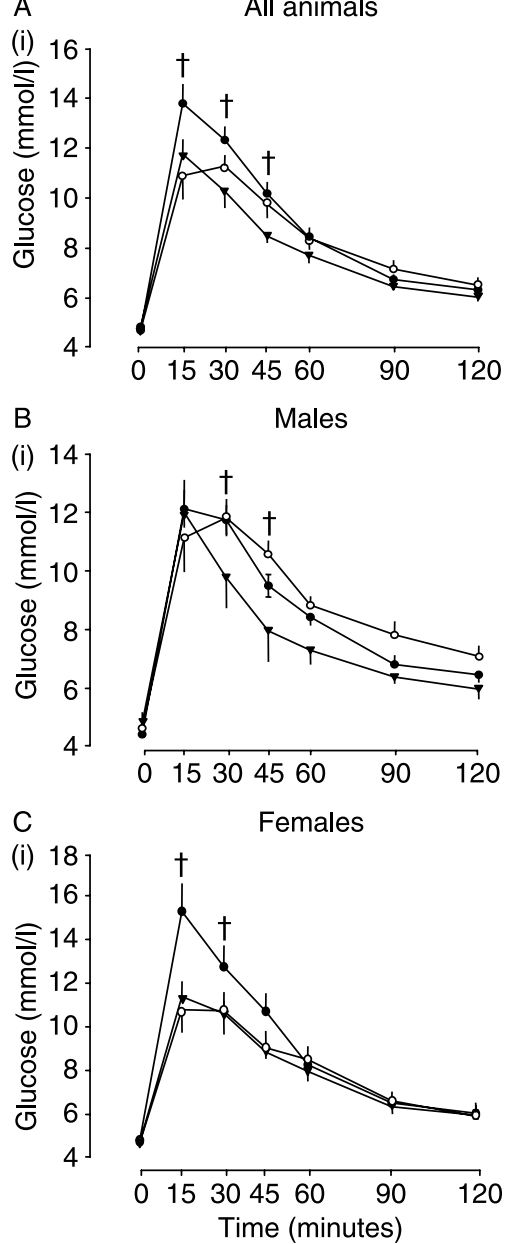

Figure 4 Mean ( \pm S.E.M.) values of (i) blood glucose concentrations with respect to time and (ii) the area under the glucose curve for $(A)$ all adult offspring irrespective of sex, (B) male offspring, and (C) female offspring of untreated control dams (Con, open circles and open columns, $n=7$ males and $n=7$ females from four dams) and of dams treated with saline (Sal, triangles and stippled columns, $n=8$ males and $n=8$ males females from four dams) or dexamethasone (Dex, closed circles and striped columns, $n=8$ males and $n=8$ females from four dams) from day 15 to day 20 of pregnancy. For (i), significant effect of maternal treatment ${ }^{\dagger} P<0 \cdot 01$ (linear mixed model with maternal treatment, sex of the offspring, and time as factors, where appropriate). For (ii), columns with different letters are significantly different from each other $(P<0 \cdot 01)$, while columns sharing common letters are not significantly different from each other $(P>0 \cdot 05$, linear mixed model).

have differential effects on the growth and glucose metabolism of their offspring. These effects depend, in part, on the age and sex of the offspring and are related to fat deposition and the plasma concentrations of insulin, leptin, and corticosterone in the adults. Compared with untreated controls, maternal dex treatment reduced offspring body weight in utero and at birth, whereas the stress associated with maternal saline injection did not. Both treatments reduced pup body weight during suckling and for 4 weeks after weaning, but only dex treatment led to a significantly higher postweaning FGR. The two treatments also had different effects on hepatic glucogenic capacity, particularly in fetal and early postnatal life. Furthermore, relative to controls, adult glucose tolerance was improved by prenatal stress in the males but not in the females, while it was impaired in the female offspring but not in the male offspring of the dex-treated dams. Since maternal corticosterone concentrations were elevated in the saline-injected dams relative to the other two groups, the current findings suggest that overexposure to both natural and synthetic glucocorticoids has programming effects in utero, but that their specific actions differ in a sex-linked manner in the rats. Thus, natural glucocorticoids may not entirely mimic the programming effects of synthetic glucocorticoids as shown previously in adult sheep overexposed to glucocorticoids in utero during early gestation (Moritz et al. 2005).

\section{Growth and body composition}

Dex administration reduced maternal weight gain during the period of treatment and restricted prenatal and early postnatal growth of the pups in line with previous findings in rats treated with glucocorticoids during late pregnancy by injection, ingestion, and continuous infusion (Smith \& Waddell 2000, Sugden et al. 2001, Swolin-Eide et al. 2002, Scheepens et al. 2003, O'Regan et al. 2004, Woods 2006). The reduced maternal weight gain during both dex and saline treatments in the current study was due, in part, to the reduced weight of the fetuses and placentas (not shown), and may also have reflected the differences in concentration of corticosterone and other stress hormones associated with injection, such as the catecholamines. It was not related to decreased food intake. However, reduced growth of maternal tissues, such as the mammary glands, may have compromised lactation relative to untreated dams, leading to lower pup weights during suckling. When the lactational constraint was lifted at weaning, there was a period of catch-up growth in both treated groups so that body weight was not significantly different between offspring of treated and untreated dams from 8 weeks of postnatal age. Similar catch-up growth after weaning has been observed in previous studies of maternal restraint stress and dex treatment during rat pregnancy (O'Regan et al. 2004, 2008, D'mello \& Lin 2006). Indeed, the degree of catch-up growth appears to depend on litter size and is complete when litters are culled to eight pups, as in the present study, but not with larger pup numbers (O'Regan et al. 2004, 2008, Wyrwoll et al. 2006, 2008). Taken together, these observations suggest that nutritional constraint during suckling may contribute to the adult metabolic outcomes associated with prenatal stress and dex exposure.

The changes in prenatal and early postnatal growth induced by maternal dex and saline treatments were accompanied by altered fat distribution in the adult offspring. In the dextreated group, the amount of excised peritoneal and perirenal fat relative to total body weight was reduced, particularly in 
the males, consistent with the reduction in epididymal fat mass observed previously in adult males exposed to dex in utero (Sugden et al. 2001, Cleasby et al. 2003). However, relative to untreated controls, there was no apparent effect of dex treatment on fat content of the abdominal region measured by DEXA scanning. The disparity between the fat content measured gravimetrically and that measured by DEXA scanning in the present study suggests that fat distribution in the adult offspring is altered by maternal dex treatment with a decrease in peritoneal and perirenal fat and an increase in subcutaneous and other abdominal fat deposits relative to untreated controls. In contrast, prenatal stress induced by maternal saline injection reduced adult DEXA fat content compared with controls in parallel with combined peritoneal and perirenal fat weight, again to a greater extent in males than in females. Offspring of the saline-treated dams, therefore, appear to have a more proportionate reduction in adiposity than that observed in the dex-treated group. However, there was no influence of maternal treatment on plasma leptin concentrations in the adult offspring, despite the differences in fat deposition and the positive correlation observed between DEXA fat content and plasma leptin levels when all the data were combined regardless of maternal treatment or sex of the offspring. These findings are consistent with previous observations that postnatal hyperleptinemia does not develop in rats exposed prenatally to dex until at least 3 months after birth irrespective of the maternal route of steroid administration (Sugden et al. 2001, Cleasby et al. 2003, Wyrwoll et al. 2006). Differences in adult body fat content and distribution programmed in utero may, therefore, precede changes in circulating leptin concentrations in the rats.

The explanation for the greater sensitivity of males than of females to treatment-induced changes in fat deposition remains unclear, but it may be related to the higher fat content of the males overall. The percentage fat content of the adult male and female rats measured by DEXA scanning in the current study was similar to those reported previously for 6-month-old Wistar rats using the same methodology (Wyrwoll et al. 2006). When all the current data were combined, fat content measured by DEXA scanning was inversely related to the plasma corticosterone concentrations. Since corticosterone tends to mobilize fat stores during stressful conditions (Bouchard et al. 1993), males may have more fat than females between 14 and 15 weeks of age because they have lower corticosterone concentrations. Males also had higher levels of insulin than females in the present study, which would tend to favor fat deposition, although insulin concentrations were not directly correlated to the DEXA fat content of the adult offspring in either sex alone or when the data from both sexes were combined. The higher concentrations of insulin and leptin and lower concentrations of corticosterone observed in males than in females between 14 and 15 weeks in the present study are consistent with previous findings of adult rats at older ages (Sugden et al. 2001, Wyrwoll et al. 2006, 2008).

\section{Hepatic glucogenic capacity}

Both maternal treatments altered the hepatic glucogenic capacity of male and female offspring relative to untreated controls. The most pronounced effects of treatment were observed in the fetuses, which suggest that hepatic glucogenic capacity in utero is glucocorticoid sensitive in rats as occurs in other species near term (Fowden et al. 1998). At birth, hepatic glycogen content and G6Pase activity were similar in the three groups of neonates, while hepatic PEPCK activity was lower in the newborn pups of the saline-treated dams than in the other two groups of neonates. In the salinetreated group, daily exposure to raised corticosterone concentrations via a maternal stress response to injection may have delayed the activation of the fetal HPA axis. In contrast, in the dex-treated group, withdrawal of the more potent synthetic steroid may have lifted negative feedback on the maternal and fetal HPA axes and allowed a rapid rebound in natural corticosterone concentrations (Fowden et al. 1998). At weaning, the effects of maternal treatment with dex, but not with saline, were still evident with raised PEPCK activity, but by between 14 and 15 weeks, neither treatment had a significant influence on the hepatic glucogenic capacity relative to untreated controls. These observations suggest that changes in PEPCK activity programmed in utero are more evident at times of major changes in nutrition at birth and weaning. However, hepatic PEPCK activity was higher in the adult offspring of the dex-treated dams than in those of the saline-treated dams as reported previously for offspring of dex- and vehicle-injected dams (Nyirenda et al. 1998, 2006). This glucocorticoid programmed upregulation of adult hepatic PEPCK activity is associated with increased expression of hepatocyte nuclear factor 4, a known transcription factor for the Pck2 promoter (Nyirenda et al. 2006). In the current study, the difference in hepatic PEPCK activity between offspring of the saline- and dex-treated dams was observed in both adult males and females, whereas previously, this effect was observed only in males (Nyirenda et al. 1998, O'Regan et al. 2004). This discrepancy between studies may be due to differences in offspring age or dose of dex administered to their dams (Nyirenda et al. 1998, 2006, O'Regan et al. 2004). Overall, the current findings suggest that the hepatic glycogenolytic and gluconeogenic pathways have differential sensitivities to programming in utero, and that hepatic PEPCK activity is particularly prone to control by the developmental environment.

\section{Glucose metabolism}

Neither maternal treatment affected basal glucose concentrations at any age studied. In previous studies, maternal restraint stress has been shown to elevate fasting glucose concentrations in adult male offspring at 6 and 24 months but not at 3-4 months of postnatal age (Vallée et al. 1996, Lesage et al. 2004, D'mello \& Lin 2006). Similarly, maternal dex treatment is associated with fasting hyperglycemia in 
6-month-old male offspring in some but not in all previous studies depending on the route of maternal administration (Sugden et al. 2001, Drake et al. 2004, O'Regan et al. 2004). In the current study, both treatments influenced glucose tolerance of the adult offspring, although in different ways depending on sex of the offspring. In males, maternal saline injection improved glucose tolerance, measured as AUGC, while maternal dex treatment had little apparent effect on this measurement relative to untreated controls. The differing AUGC with treatment was largely due to differences in glucose clearance as the peak glucose concentration was similar in the three male groups. The cause of these differences in clearance remains unclear, but glucose tolerance was best in the saline-treated group, which had the highest plasma insulin concentration. There was also a positive correlation between male AUGC and DEXA fat content, which suggests that adipose tissue-derived factors reducing insulin sensitivity may also have contributed to the relative glucose intolerance of the fatter male groups (Stocker et al. 2005). In contrast, female glucose tolerance was unaffected by maternal saline injection, but it was impaired by dex treatment relative to untreated controls. The difference between female AUCG with treatment was primarily due to increased peak glucose concentrations in the dex-treated group. Since female fat content was unaffected by treatment, the glucose intolerance of females that were exposed to dex in utero may reflect poor first-phase insulin secretion or an increased rate of glucose absorption from the peritoneal cavity relative to the other groups. In addition, there may be sex-linked differences in the response of glucoregulatory hormones, such as insulin, corticosterone, and catecholamines, to i.p. injection of glucose, which contribute to the observed differences in adult glucose tolerance with maternal treatment.

In the present study, glucose tolerance was worse in the dex-treated group than in the saline-treated group in both sexes. The reason for this treatment difference appeared to depend on sex of the offspring with improved glucose clearance in the saline-treated group of males but impaired glucose tolerance in the dex-treated group of females relative to the untreated controls. In addition, hepatic PEPCK activity was highest in adult offspring of the dex-treated dams, irrespective of sex. Using an oral glucose tolerance test, a similar impairment of glucose tolerance coupled with high hepatic PEPCK activities has been observed in 6-month-old male offspring but not in female offspring of rats given dex by the same route for the same period of gestation as in the current study compared with vehicle-treated controls (O'Regan et al. 2004). The reasons for the differing responses of adult females prenatally exposed to dex between the current and earlier studies are unclear, but they may be related to differences in dex dose, vehicle composition, route of glucose administration, stage of estrous cycle, or offspring age at study (Nyirenda et al. 1998, O’Regan et al. 2004). However, taken together, these studies suggest that enhanced hepatic glucose production may also be a contributory factor to the glucose intolerance of adult rats prenatally exposed to dex (Seckl 2008). Abnormalities in hepatic glucose production associated with elevated PEPCK activity have also been observed in male offspring of rats that were deprived of protein during pregnancy (Burns et al. 1997, Desai et al. 1997).

Overall, the differences in intrauterine programming observed between maternal dex and saline treatments appear to be related, in part, to differences in body weight in fetal and early postnatal life and to altered fat content and distribution in the adult offspring. These differences in programming have implications for evaluating studies using control procedures that raise endogenous glucocorticoid concentrations. This may explain, in part, the differences in glucocorticocoid programming observed in previous studies using differing routes of steroid administration and, hence, control procedures (Sugden et al. 2001, Cleasby et al. 2003, Wyrwoll et al. 2006). However, since glucocorticoids alter prenatal development of many tissues involved in postnatal glucoregulation (Fowden et al. 1998), these hormones may be a common factor linking poor fetal growth during suboptimal conditions to developmental programming of postnatal glucose metabolism, particularly as many of the natural and experimental conditions leading to IUGR increase glucocorticoid exposure in utero.

\section{Declaration of interest}

The authors declare that there is no conflict of interest that would prejudice the impartiality of the research reported.

\section{Funding}

This research was funded by the Gates Cambridge Trust.

\section{Acknowledgements}

The authors would like to thank Prof. Gordon Smith for his help with the statistical analyses, the staff of the animal house for their daily care of the animals, and the Gates Cambridge Trust for funding the PhD studies of KLF.

\section{References}

Barbazanges A, Piazza PV, Le Moal M \& Maccari S 1996 Maternal glucocorticoid secretion mediates long-term effects of prenatal stress. Journal of Neuroscience 16 3943-3949.

Barker DJP 1994 Mothers, Babies and Disease in Later Life., London: BMJ Publishing Group.

Bouchard C, Després JP \& Maurèque P 1993 Genetic and non-genetic determinates of regional fat distribution. Endocrine Reviews 14 72-93.

Burns SP, Desai M, Cohen RD, Hales CN, Iles RA, Germain JP, Going TC \& Bailey RA 1997 Gluconeogenesis, glucose handling and structural changes in livers of adult offspring of rats partially deprived of protein during pregnancy and lactation. Journal of Clinical Investigation $\mathbf{1 0 0}$ 1768-1774. 
Cleasby ME, Kelly PAT, Walker BR \& Seckl JR 2003 Programming of rat muscle and fat metabolism by in utero overexposure to glucocorticoids. Endocrinology 144 999-1007.

Desai M, Byrne CD, Meeran K, Hartenz ND, Bloom SR \& Hales CN 1997 Regulation of hepatic enzymes and insulin levels in the offspring of rat dams fed a reduced protein diet. American Journal of Physiology 273 G899-G904.

D'mello AP \& Lin Y 2006 Effects of maternal immobilization stress on birthweight and glucose homeostasis in the offspring. Psychoneuroendocrinology 31 395-406.

Drake AJ, Walker BR \& Seckl JR 2004 Intergenerational consequences of fetal programming: non-genomic mechanisms of the interaction of low birth weight and cardiovascular risk. Journal of Endocrinology 180 1-16.

Folch J, Lees M \& Sloane Stanley GH 1957 A simple method for the isolation and purification of total lipids from animal tissue. Journal of Biological Chemistry 226 497-509.

Fowden AL, Mijovic J \& Silver M 1993 The effects of cortisol on hepatic and renal gluconeogenic enzymes during late gestation. Journal of Endocrinology 137 213-222.

Fowden AL, Li J \& Forhead AJ 1998 Glucocorticoids and the preparation for life after birth: are there long term consequences of the life insurance? Proceedings of the Nutrition Society 57 113-122.

Fowden AL, Giussani DA \& Forhead AJ 2006 Intrauterine programming of physiological systems: causes and consequences. Physiology 21 29-37.

Franko KL, Giussani DA, Forhead AJ \& Fowden AL 2007 Effects of dexamethasone on the glucogenic capacity of fetal, pregnant and non-pregnant adult sheep. Journal of Endocrinology 191 67-73.

Franko KL, Forhead AJ \& Fowden AL 2009 Effects of maternal dietary manipulation during different periods of pregnancy on hepatic glucogenic capacity in fetal and pregnant rats near term. Nutrition, Metabolism, and Cardiovascular Diseases 19 555-562.

Gluckman PD, Hanson MA, Cooper C \& Thornburg KL 2008 Effect of in utero and early-life conditions on adult health and disease. New England Journal of Medicine 359 61-73.

Lesage J, Del-Favero F, Leonhardt M, Louvart H, Maccari S, Vieau D \& Darnaudery M 2004 Prenatal stress induces intrauterine growth restriction and programmes glucose intolerance and feeding behaviour disturbances in the aged rat. Journal of Endocrinology 181 291-297.

Maccari S, Darnaudery M, Morley-Fletcher S, Zuera AR, Cinque C \& Van Reeth O 2003 Prenatal stress and long term consequences: implications of glucocorticoid hormones. Neuroscience and Biobehavioral Reviews $\mathbf{2 7}$ 119-127.

McMillen IC \& Robinson JS 2005 Developmental origins of the metabolic syndrome: prediction, plasticity and programming. Physiological Reviews $\mathbf{8 5}$ 571-633.

Moritz KM, Boon WM \& Wintour EM 2005 Glucocorticoid programming of adult disease. Cell and Tissue Research 322 81-88.

Muneoka K, Mikuni M, Ogawa T, Kitera K, Kamei K, Takigawa M \& Takahashi K 1997 Prenatal dexamethasone exposure alters brain monoamine metabolism and adrenocortical responses in rat offspring. American Journal of Physiology 273 R1669-R1675.

Nyirenda MJ, Lindsay RS, Kenyon CJ, Burchell A \& Seckl JR 1998 Glucocorticoid exposure in late gestation permanently programs rat hepatic phosphoenolpyruvate carboxykinase and glucocorticoid receptor expression and causes glucose intolerance in adult offspring. Journal of Clinical Investigation 101 2174-2181.

Nyirenda MJ, Dea S, Lyons V, Chapma KE \& Seckl JR 2006 Prenatal programming of hepatocyte nuclear factor $4 \alpha$ in the rat: a key mechanism in the "foetal origins of hyperglycaemia"? Diabetologia 49 1412-1420.
O’Regan D, Kenyo CJ, Seckl JR \& Holmes MC 2004 Glucocorticoid exposure in late gestation in the rat permanently programs gender-specific differences in adult cardiovascular and metabolic physiology. American Journal of Physiology. Endocrinology and Metabolism 287 E863-E870.

O'Regan D, Kenyon CJ, Seckl JR \& Holmes MC 2008 Prenatal dexamethasone 'programmes' hypotension, but stress-induced hypertension in adult offspring. Journal of Endocrinology 196 343-352.

Poore KR \& Fowden AL 2002 The effect of birth weight on glucose tolerance in pigs at 3 and 12 months of age. Diabetologia 45 1247-1254.

Poore KR \& Fowden AL 2004 The effects of birth weight and postnatal growth patterns on fat depth and plasma leptin concentrations in juvenile and adult pigs. Journal of Physiology 558 295-304.

Scheepens A, van de Waarenburg M, van den Hore D \& Blanco CE 2003 A single course of prenatal betamethasone in the rat alters postnatal brain cell proliferation but not apoptosis. Journal of Physiology $\mathbf{5 5 2}$ 163-175.

Seckl JR 2008 Glucocorticoids, developmental 'programming' and the risk of effective dysfunction. Progress in Brain Research 167 17-34.

Smith JT \& Waddell BJ 2000 Increased fetal glucocorticoid exposure delays puberty onset in postnatal life. Endocrinology 141 2423-2428.

Stocker CJ, Arch JRS \& Cawthorne MA 2005 Fetal origins of insulin resistance and obesity. Proceedings of the Nutrition Society 64 143-151.

Sugden MC, Langdown ML, Munns MJ \& Holness MJ 2001 Maternal glucocorticoid treatment modulates placental leptin and leptin receptor expression and materno-fetal leptin physiology during late pregnancy, and elicits hypertension associated with hyperleptinaemia in the early-growth-retarded adult offspring. European Journal of Endocrinology 145 529-539.

Swolin-Eide D, Dahlgren J, Nilsson C, Albertsson Wikland K, Holmäng A \& Ohlsson C 2002 Affected skeletal growth but normal bone mineralization in rat offspring after prenatal dexamethasone exposure. Journal of Endocrinology 174 411-418.

Vallée M, Mayo W, Maccari S, Le Moal M \& Simon H 1996 Long term effects of prenatal stress and handling on metabolic parameters: relationship to corticosterone secretion response. Brain Research 712 287-292.

Ward IL \& Weisz J 1984 Differential effects of maternal stress on circulating levels of corticosterone, progrsterone and testoterone in male and female rat fetuses and their mothers. Endocrinology 114 1635-1644.

Woods L 2006 Maternal glucocorticoids and prenatal programming of hypertension. American Journal of Physiology. Regulatory, Integrative and Comparative Physiology 291 R1069-R1075.

Wyrwoll CS, Mark PJ, Mori TA, Puddey IB \& Waddell BJ 2006 Prevention of programmed hyperleptinemia and hypertension by postnatal dietary $\omega-3$ fatty acids. Endocrinology 147 599-606.

Wyrwoll CS, Mark PJ, Mori TA \& Waddell BJ 2008 Developmental programming of adult hyperinsulaemia, increased proinflammatory cytokine production, and altered skeletal muscle expression of SLC2A4 (GLUT4) and uncoupling protein 3. Journal of Endocrinology 198 571-579.

Received in final form 2 December 2009 Accepted 16 December 2009 Made available online as an Accepted Preprint 16 December 2009 